\title{
Nonlinear Dynamics of Degradation of Vallisneria Spiralis L. Chlorophyll Photoluminescence at High Excitation Levels
}

\section{OPEN ACCESS}

Citation: Vakulenko O.V., Grygorieva O.O. (2018) Nonlinear Dynamics of Degradation of Vallisneria Spiralis L. Chlorophyll Photoluminescence at High Excitation Levels. Open Science Journal 3(1)

Received: $25^{\text {th }}$ October 2018

Accepted: $28^{\text {th }}$ January 2018

Published: $15^{\text {th }}$ March 2018

Copyright:@ 2018 This is an open access article under the terms of the Creative Commons

Attribution License, which permits unrestricted use, distribution, and reproduction in any medium, provided the original author and source are credited.

Funding: The author(s) received no specific funding for this work

Competing Interests: The author have declared that no competing interests exists.

\author{
Olegh V. Vakulenko $^{1 *}$, Olesja O. Grygorieva ${ }^{2}$ \\ ${ }^{1}$ Faculty of Physics, Taras Shevchenko National University of Kyiv, Kyiv, \\ Ukraine \\ ${ }^{2}$ Educational and Scientific Centre "Institute of Biology", Taras \\ Shevchenko National University of Kyiv, Kyiv, Ukraine \\ *Corresponding author: Olegh Vakulenko: ovva37@gmail.com
}

\begin{abstract}
:
In the context of the study of the photoinhibition mechanisms of the higher water plant Vallisneria spiralis L. photosynthetic machinery, the fluorescence intensity degradation of chlorophyll $a(687 \mathrm{~nm})$ leaf fragments at photoexcitation levels from 7 to 150 $\mathrm{mW} / \mathrm{cm} 2$ on the wave length of $488 \mathrm{~nm}$, is investigated. It is shown that the luminescence efficiency decrease rate cannot be described by simple exponential or hyperbolic dependence. To explain this behavior, a kinetic model accounting for dimerization of luminescent molecules under the influence of excess lighting, is put forward. It is supposed that dimers are not capable to produce luminescence in the determined spectral area and, therefore, to transmit energy to their molecules of a photosystem and, eventually, to the reaction center. This results in a plant's photosynthetic activity decrease.
\end{abstract}

Keywords: Water plants, Photoinhibition mechanism, Fluorescence intensity, Luminescence efficiency.

\section{Introduction}

Nowadays, the fluorescence kinetics concept is associated with the chlorophyll fluorescence induction curve [1], the form of which is very sensitive to the changes occurring in the photosynthetic machinery in the course of adaptation to different 
environment conditions. This fact became a basis of wide use of the corresponding method in photosynthesis studies. It was shown by H. Kautsky and A. Hirsch [2] that illumination of the plants being previously adapted for darkness, with the blue actinic light leads to the sharp growth of red fluorescence of a chlorophyll in the first seconds from the moment of illumination switching, and after that the fluorescence intensity gradually decreases to some stationary level. The described phenomenon received the name of the Kautsky effect.

The purpose of our work is a study of the properties of this "stationary level" of fluorescence, in order to receive new information on the nature of photo chemical luminescence quenching and, respectively, photo inhibition of the photosynthetic machinery of the water plant Vallisneria spiralis L.

Photo inhibition is light-induced reduction in the photosynthetic capacity of a plant, alga, or cyano bacterium. In 1956, B. Kok [3] first used the term "photo inhibition", although the detrimental effects of excessive light exposure on plant photosynthesis have been known much earlier[4]. Photo system II (PSII) is more sensitive to light than the rest of the photosynthetic machinery, and most researchers define the term as light-induced damage to PSII [3,5-8]. Photo inhibition is also used in a wider sense as dynamic photo inhibition, to describe all reactions that decrease the efficiency of photosynthesis when plants are exposed to light. In living organisms, the photo inhibited PSII centers are continuously repaired via degradation and synthesis of the D1 protein of the photosynthetic reaction center of PSII.

Various hypo theses of molecular mechanisms of PSII photo inhibition were formulated in [9]. Essentially, photo inhibition is represented by a series of photochemical reactions that suppress this or that activity of PSII, but there is no consensus about the nature of these reactions. The water oxidizing complex (oxygen-evolving manganese cluster) is usually the first to lose its activity [9-12].

The PSII photo damage process which can be monitored in the absence of the PSII repair process, is strongly related to the incident light intensity [13-16], where the photo damage degree is directly proportional to the light intensity. Also, according to the mathematical model [17], the PSII complexes photo in activation resulting from excessive lighting, is a first order reaction that can be described by the linear differential equation which explains linear dependence of the photo inhibition effect on the light intensity and predetermines exponential temporary dependence of this influence.

However, a distinctively nonlinear dependence of photoluminescence (PL) degradation of the water plant Vallisneria spiralis L. on the excitation light intensity was revealed in [18] that induced us to study this question in more detail. In this article, we a put forward kinetic model accounting for dimerization of luminescent molecules under the influence of excess lighting. We argue that the dimers are not capable to produce lumine scence in the determined spectral area and to transmit energy to other molecules of a photo system and, eventually, to the reaction center. This results in a plant's photo synthetic activity decrease. 


\section{Materials and Methods}

As in the previous works [18-20], the object of study was PL of chlorophyll $a$ of the leaf fragments of the higher water plant Vallisneria spiralis L. The lumine scence was excited by radiation with the wave length of $488 \mathrm{~nm}$ of the argon laser of continuous action LGN-402, the excitation density varied in the rangefrom 7 to $150 \mathrm{~mW} / \mathrm{cm} 2$. The flare level was controlled by the power meter at the beginning and at the end of each experiment, where disarrangement in indications was no more than $3 \%$. The spectrum of chlorophyll PL consisted of two bands: the dominating line with a maximum at $\lambda=687 \mathrm{~nm}$ and the wider band in the area of $\lambda=740 \mathrm{~nm}$. Major fluorescence band at $687 \mathrm{~nm}$ originates mostly in the PSII antenna complexes, whereas the band of $\lambda=740 \mathrm{~nm}$ is determined mostly by the PSI antenna complexes [21]. The degradation was investigated at the room temperature at the spectrum maximum $(687 \mathrm{~nm})$. Time reckoning was conducted from the flare beginning moment, the interval between experimental points was 0.3 seconds. As only the decrease stage of the luminescence intensity was taken in to account, the first 7 seconds of measurement were excluded from consideration.

\section{Results}

In the course of our researches, dynamic curves of PL intensity decrease at different excitation levels were obtained. The maximum power density, as mentioned above, was $150 \mathrm{~mW} / \mathrm{cm} 2$. The corresponding curves reduced to unit initial intensity, are plotted in Fig. 1.

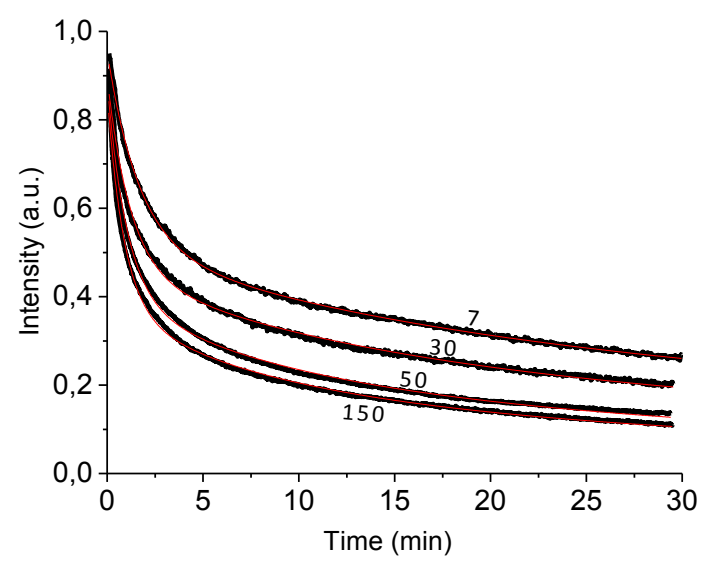

Figure 1. Kinetics of photo luminescence degradation of Vallisneria spiralis L. chlorophyll at different levels of excitation. Experimental (black) and calculated from the equation(1)(red dotted line) degradation dynamics of PL at various values of excitation density $(\mathrm{mW} / \mathrm{cm} 2)$. The curve is normalized to the calculated intensity at $\mathrm{t}=0$. 
It can be seen that, expectedly, the degradation is accelerated as the flare intensity increases. However, at sufficiently high excitation levels, the form of curves ceases to depend on the pumping power. The fact that the PL degradation rate depends on excitation intensity, manifests that the corresponding curve cannot be presented by an exponent only or by any combination of exponents.

The experimental dependences were approximated by means of the Origin 9.1 program.

As it appeared, the results presented on Fig. 1, may be well described by the following formula representing the sum of an exponent and a hyperbola:

$I(t)=\operatorname{cexp}(-a t)+\frac{d}{1+b t}$,

where a, b, c, d are the adjustment parameters, the values of which are presented in Table1: c, d - dimensionless parameters; $a, b$ - probability of linear and quadratic recombination, respectively. The average lifetime of a molecule with linear recombination is $\tau=1 / a$.

Table 1: The values of the parameters in the formula (1) used for plotting the curves in Fig. 1.

\begin{tabular}{|l|c|c|c|c|c|}
\hline Caption & $\mathrm{mW} / \mathrm{cm}^{2}$ & $\mathrm{c}$ & $\tau, \min$ & $\mathrm{D}$ & $\mathrm{b}, \mathrm{min}^{-1}$ \\
\hline 7 & 7 & 0.33 & 1.6 & 0.39 & 0.033 \\
\hline 30 & 30 & 0.33 & 1.2 & 0.36 & 0.047 \\
\hline 50 & 50 & 0.38 & 1.00 & 0.34 & 0.077 \\
\hline 150 & 150 & 0.39 & 0.90 & 0.31 & 0.086 \\
\hline
\end{tabular}

One should pay attention to one nontrivial result of this experiment. In our conditions, the light intensity is 2-3 orders of magnitude higher than that of sun light (0.15 $\mathrm{mW} / \mathrm{cm}^{\wedge} 2 \bullet \mathrm{nm}$ at 450-500 nm.) However, in the wide region, changes in the intensity of $\operatorname{excitation}(I=7-150 \mathrm{~mW} / \mathrm{cm} \wedge 2$ at $488 \mathrm{~nm})$, the kinetics of the PL degradation can be well described by the same formula (1). Nevertheless, we are confused by the detected dependence of $\tau(I)$. But the behavior of $b(I)$ can be partially explained by the following theory, which will serve for justification of the empirical formula (1).

\section{Theoretical model}

In the previous article [18], an at tempt was made to represent a degradation curve as the sum of two hyperbolas. However, our further studies showed that such approach should be considered as the first formal approximation which does not reveal physical essence of luminescence degradation processes of the studied 
biological objects. Further scrupulousre searches showed a need to revise the kinetic equations.

We will briefly consider basic provisions of this new theory.

Let $N$ be the number of radiating centers (in this case, chlorophyll molecules) of a luminescent system. At the time $t, N_{0}$ of them reside in the ground state and $N^{*}$ are excited. We will write down the simplest kinetic equation:

$$
\frac{d N^{*}}{d t}=g N_{0}-\frac{N^{*}}{\tau}-\beta N^{*}-\gamma\left(N^{*}\right)^{2}
$$

Here $g$ is the probability of the molecule's transition from the ground to an excited state (it is proportional to the exciting light intensity), $\tau$ is the radiative transition time. The instant luminescence intensity is proportional to the quantity of molecules passing from the excited to the ground state in a time unit, that is to the term $N^{*} / \tau$. The coefficient $\beta$ is the probability of the linear radiation less recombination.

The last addendum in the right part of the second equation describes the creation rate of radiation less dimers. It is known [21] that dimers can form dyes under the influence of light. We assume that the chlorophyll dimers are not capable to luminescence, at least, in the studied spectral range. Here $\gamma$ is the coefficient of dimerization probability determining its rate. According to the research [18], degradation is partially reversible, but for simplicity we will omit here the corresponding term.

As $g N_{0}, \frac{N^{*}}{\tau}>>\beta N^{*}, \gamma\left(N^{*}\right)^{2}$ in (2), it is possible to allocate here the fast and slow subsystems. Really, the kinetic processes of the chlorophyll fluorescence are significantly faster than the degradation ones: the recombinational life time $\tau$ of the excited state is known to be about $10^{-8} \mathrm{~s}$ [22] while the characteristic degradation times make $10-10^{3} \mathrm{~s}[18,23]$. So we will take it further that the reduction of the number of the radiativere combination centers occurs much slower than the excitation-recombination does. We will apply here the adiabatic approximation method. The given problem is being solved in two stages: at first the movement of the fast subsystem at the fixed parameters of the slow one is considered, and then the movement of the last is accounted for.

We will write down for the fast subsystem:

$$
\frac{d N^{*}}{d t}=g N_{0}-\frac{N^{*}}{\tau} \text {. }
$$

We obtain from (3) the solution for stationary conditions:

$$
N_{s t}^{*}=g N_{0} \tau \text {. }
$$

The quantity $N^{*}$ st is a slow function of time, which can be obtained from the equation

$$
\frac{d N_{s t}^{*}}{d t}=-\beta N_{s t}^{*}-\gamma\left(N_{s t}^{*}\right)^{2} .
$$


To obtain the solution of this equation in the form of (1), it is necessary to assume that the linear and quadratic radiationless recombinations are in dependent processes. It is possible to write in this case:

$$
\begin{aligned}
& \frac{d N_{1 s t}^{*}}{d t}=-\beta N_{1_{s t}}^{*}, \\
& \frac{d N_{2 s t}^{*}}{d t}=-\gamma\left(N_{2 s t}^{*}\right)^{2}, \\
& N_{s t}^{*}=N_{1 s t}^{*}+N_{2 s t}^{*} .
\end{aligned}
$$

After solving this system of equations (6) - (8), we obtain:

$$
N_{s t}^{*}(t)=N_{1 s t}^{*}(0) \exp (-\beta t)+\frac{N_{2 s t}^{*}(0)}{1+\gamma N_{2 s t}^{*}(0) t}
$$

After replacing: $N_{1 s t}^{*}(0)=\mathrm{c}, \beta=a, N_{2 s t}^{*}(0)=d, \gamma N_{2 s t}^{*}(0)=b-$ we get the equation (1).

The formula (9) obtained coincides with the empirical formula and explains the growth of $b$ by an increase in $I$ (see Table 1 ). However, in order to explain $\tau$ (I), the kinetic equation (2) becomes complicated. But this is a separate task.

It was already noted that the excitation-recombination processes happen much faster than dimerization. Thus as a calculated initial value $N^{*}{ }_{s t}(0)$ in (4), it is expedient to consider the quantity obtained from the formula $N_{0}=N-N^{*}$. In this case

$$
N_{\mathrm{st}}^{*}(0)=\frac{g N}{g+1 / \tau} .
$$

Let us note that at an intensive excitation $(g>>1 / \tau), N^{*}{ }_{s t}(0) \rightarrow N$, and for a weak excitation $(\mathrm{g}<<1 / \tau), N^{*}{ }_{s t}(0) \approx g \tau N$. Thus, the maximum luminescence intensity $I_{0}$ has to be proportional to the excitation intensity at low levels of the last, being saturated at high levels. It is seen from Fig. 2 that the signal amplitude $I_{0}$ increases from zero with the excitation level increase, in accordance to $(10)$.

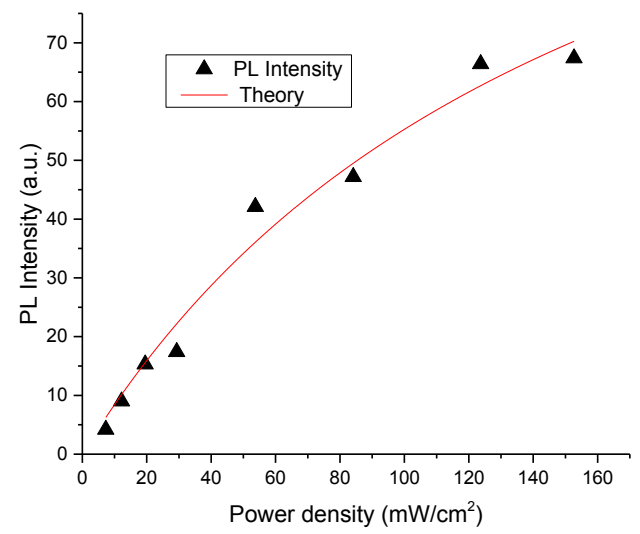

Figure. 2. Photo luminescence intensity of chlorophyll $a$ of the leaf fragments of the higher water plant Vallisneria spiralis L. at different excitation levels. The points represent the experiment, the continuous curve represents calculation by

(10) with the use of the Origin 9.1 program. 
Our method also correctly describes the experimental results obtained in [24] where the initial site of photo damage to PSII under visible light using PSII membrane fragments isolated from spinach leaves was examined. It appears that these results are well described by the sum of two exponents (Fig. 3):

$$
Y=c \cdot \exp (-a t)+d \cdot \exp (-b t)
$$

Where $Y$ is the PSII activity, and $a, d, c, d$ are constants

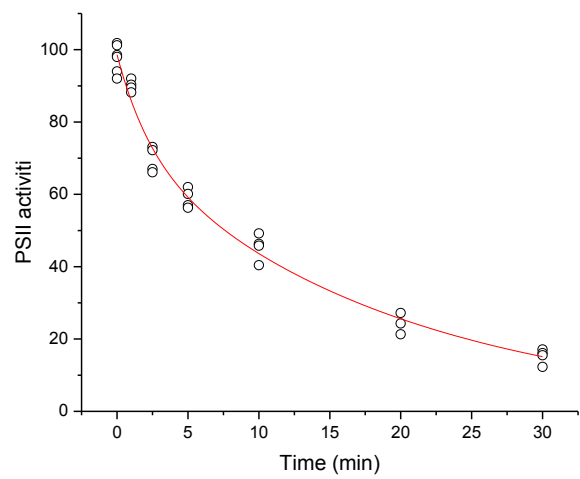

Figure 3: Photo damage to PSII by visible light [24]. The total PSII activity was then measured by monitoring the light-dependent oxygen production. The red curve is calculated by formula (11) with the following parameters: $a=0.44$

$$
\min ^{-1}, b=0.053 \min ^{-1}, c=25.3, d=73.3
$$

ThisdependenceisdescribedbythelinearkineticequationforthePSIIactivity that, to our mind, may have the following appearance:

$$
\frac{d y}{d t}=G-\frac{y}{\tau}-a y-b y,
$$

Where $y$ is the concentration of photo excited active centers PSII, $G$ - is the photo generation rate of such centers, $y / \tau$ is the rate of the centers deactivation (exclusion from the photo synthesis process) resulting from PL or thermal dissipation of the excitation energy (non-photo chemical PL quenching), and $a$ and $b$ are the probabilities of two independent photochemical processes. The decay times of these processes differ by almost an order of magnitude: $\tau_{\mathrm{a}}=1 / \mathrm{a}=$ $2.3 \mathrm{~min}, \tau_{\mathrm{b}}=1 / \mathrm{b}=19 \mathrm{~min}$.

Equation (12) satisfies the inequality: $\tau_{a}, \tau_{b}>>\tau$. Thus, the adiabatic approach described above can be applied here. Assuming an independent character of the processes ay and i, we arrive at (11).

\section{Discussion}

So, it is possible to conclude that the proposed kinetic equation (2) combined with an unfamiliar method of its solution, describes well the photo luminescence degradation dynamics of a leaf of the higher water plant Vallisneria spiralis L. at 
excessive lighting. Two types of the PL degradation mechanisms follow from here: linear and square ones. The last, in our opinion, is caused by photo dimerization of chlorophyll molecules which happens in the light-harvest in gantenn a complexes area. The dimers lose their ability to luminesce in the studied spectrum range and, most importantly, to support migration of the excitation energy to the reaction centers of photo systems.

The linear par tof the PL degradation which correlates with the photo synthesis efficiency decrease [see 13], is rather widely discussed in literature and does not need additional arguments. It is important for us that, as already mentioned, the water oxidizing complex is in this case the first to lose its activity [9-12]. It follows from here that the linear and nonlinear processes are spatially separated and therefore independent, that was supposed during solution of the equation (5).

The kinetics of the PSII photo damage by the visible light [see 24] described here by the sum of two exponents (see Fig. 3), may evidence in favor of the twostep photo damage model [see 11].

We notice that the experimental results presented in Fig. 1, may be successfully described by an allometric hyperbola also [20], and results of Fig. 3 may be described by an allometric exponent, i.e. Kohlrausch function [25]. However, the description accepted in this work, is more in formative and clear.

We note that similar researches were conducted earlier [23], where an exponential dynamics of PL degradation was observed. There the time constants for different PL bands are positioned as identical, though the radiation spectrum change with time is not explained. The seeming contradiction of results of this work with our findings may be explained by different experiment conditions. First of all, the authors of [23] used a less powerful source of excitation. Secondly observed the PL induction for a rather short time (up to $200 \mathrm{~s}$ ). Next, it is difficult to judge from their work about fluorescence excitation intensity which was carried out at the wave length of $407 \mathrm{~nm}$, i.e.in the absorption band of the chlorophyll $a$. In our case $(488 \mathrm{~nm})$, the PL excitation arose mainly through chlorophyll $b$ [26], overwhelming part of which is present in the composition of the light harvesting complexes (LHC) of the photo system II and is not met in the complexes of the reaction centers [27]. The last circumstance allows one to assume that the difference in the luminescence degradation dynamics can be conditioned by different channels of its excitation. Besides, it is noteworthy that in the mentioned work, the research object was different (acacialeaves).

\section{Conclusions}

The fluorescence intensity of the chlorophyll $a(\lambda=687 \mathrm{~nm})$ of the leaf fragments of the higher water plant Vallisneria spiralis L. displays sub linear dependence on the level of laser excitation $\left(7-150 \mathrm{~mW} / \mathrm{cm}^{2}\right)$ at the wave length of $488 \mathrm{~nm}$. This is connected with the fact that the degradation rate of the $\mathrm{PL}$ intensity super linearly grows as the excitation power increases. This dependence can be obtained within the proposed theory that provides for a possibility of the photo excited chlorophyll molecules to associate into dimers which do not take part in the luminescence process. 
Absorption of the light quanta by the chlorophyll molecule softhe antenna complex FSII is associated with the excitation energy migration to the reaction center (P680). In our opinion, in the course of this migration, the adjacent molecules may also be excited. These molecules form a photo dimer that results in a decrease of the flux of the excitation energy migration to the reaction center and, therefore, a in a decrease of the PL quantum output.

As loss of activity of the photo system II correlates with reduction of the fluorescence coefficient [13], it is possible to speak here about correlation of the PL degradation with FSII photo inhibition.

We notice that in the classical representation, the photo dimers are usually formed during interaction of one excited molecule with another unexcited molecule [21], however the logic of the quadratic recombination of equation (5) prompts that in our case, the dimerization of two photo excited chlorophyll molecules occurs.

Thus, nonlinar decrease of the luminescence intensity takes place because of the LHC depletion and deterioration of excitation transfer to the reaction center which, as is known, is also the luminescence center in the photo system II [27]. The leaf "decoloration" connected with this effect, is observed by a naked eye.

\section{References:}

1. Lazár D. Chlorophyll a fluorescence induction. Biochim. Biophys. Acta 1412, 1-28 (1999).

2. Kautsky H., Hirsch A. Neue Versuche zur Kohlensaure-assimilation. Narurwiss. 119, 964-964 (1931).

3. Kok B. On inhibition of photosynthesis by intense light. Biochim Biophys Acta 21: 234-244 (1956).

4. Ball R., Wild A. New trends in photobiology: History of photoinhibition research. J. Photochem. Photobiol. B: BioL 20 79-85, (1993).

5. Kyle D. J. The biochemical basis of photoinhibition of Photosystem II. In: Kyle DJ, Osmond CB and Arntzen CJ (eds) Photoinhibition, pp 197-226. Elsevier Publishers, Amsterdam (1987).

6. Barber J. Photoinactivation of the isolated Photosystem II reaction centre and its prevention. In: Douglas RH, Moan J and Ronto G (eds) Light Biology and Medicine, pp 21-22. Plenum Press, New York (1991).

7. Barber J. and Andersson B. Too much of a good thing: light can be good and bad for photosynthesis. Trends Biochem Sci 17: 61-66 (1992).

8. Prasil O., Adir N. and Ohad I. Dynamics of Photosystem II: mechanism of photoinhibition and recovery processes. In: Barber J (ed) The Photosystems: Structure, Function and Molecular Biology, pp 295-348. Elsevier Publishers, Amsterdam (1992).

9. Tyystjärvi E. «Photoinhibition of Photosystem II and photodamage of the oxygen-evolving manganese cluster». Coordination Chemistry Reviews 252 (3-4): 361-376 (2008).

10. Hakala M., Tuominen I., Keränen M., Tyystjärvi T. \& Tyystjärvi E. Evidence for the role of the oxygen-evolving manganese complex in photoinhibition of Photosystem II. Biochimica et Biophysica Acta (BBA) - Bioenergetics 1706 (1-2): 68-80 (2005).

11. Ohnishi N., Allakhverdiev S. I., Takahashi S., Higashi S., Watanabe M., Nishiyama Y. \& Murata N. Two-Step Mechanism of Photodamage to Photosystem II: Step 1 Occurs at the Oxygen-Evolving Complex and Step 2 Occurs at the Photochemical Reaction Center. Biochemistry 44 (23): 8494-8499 (2005).

12. Krieger-Liszkay A., Fufezan C. \& Trebst A. Singlet oxygen production in photosystem II and related protection mechanism. Photosynthesis Research 98 (1-3): 551-564 (2008).

13. Tyystjärvi E. \& Aro E. M. The rate constant of photoinhibition, measured in lincomycin-treated leaves, is directly proportional to light intensity. Proc. Natl. Acad. Sci. USA 93, 2213-2218 (1996).

14. Mattoo A. K., Hoffman-Falk H., Marder J. B. \& Edelman M. Regulation of protein metabolism: coupling of photosynthetic electron transport to in vivo degradation of the rapidly metabolized 
32-kilodalton protein of the chloroplast membranes. Proc. Natl. Acad. Sci. USA 81, 1380-1384 (1984).

15. Nishiyama Y. et al. Oxidative stress inhibits the repair of photodamage to the photosynthetic machinery. EMBO J. 20, 5587-5594 (2001).

16. Allakhverdiev S. I. \& Murata N. Environmental stress inhibits the synthesis de novo of proteins involved in the photodamage-repair cycle of photosystem II in Synechocystis sp PCC 6803. Biochim. Biophys. Acta 1657, 23-32 (2004).

17. Wünschman G. and Brand J. J. Rapid turnover of a component required for photosynthesis explains temperature dependence and kinetics of photoinhibition in a cyanobacterium, Synechococcus 6301. Planta 186: 426-433 (1992).

18. Vakulenko O., Grygorieva O., Dacenko O. Degradation of chlorophyll luminescence in plants. Ukr. J. Phys. 57, 256-259 (2012).

19. Vakulenko O., Grygorieva O., Dacenko O., Zujev V. Degradation dynamics of low-temperature chlorophyll photoluminescence in plants. In: Proccedings of the 11th International Young Scientists Conference Optics and High Technology Material Science SPO-2010, Kyiv, page 173.

20. Dacenko O. I., Vakulenko O. V., Gryrorieva O. O. Allometric dynamics of chlorophyll photoluminescence degradation at strong excitation. In: Proccedings of the 17th International Young Scientists Conference Optics and High Technology Material Science SPO-2016, Kyiv, page 59.

21. Terenin A. N. Photonics of Dye Molecules and Related Organic Compounds. Leningrad: Nauka, 616 p. (1967).

22. Perrin F. La fluorescence des solutions. Induction moléculaire - Polarisation et durée d'émission. Photochimie. Ann. phys. (Paris) 12, 169-275 (1929).

23. Borisov B. A, Bykov O. D. Spectral changes in the fluorescence of chlorophyll during photosynthesis induction. Optics and Spectroscopy 104, 186-189 (2008).

24. Zavafer A., Cheah M. H., Hillier W., Chow W. S., Takahashi S. Photodamage to the oxygen evolving complex of photosystem II by visible light. Scientific Reports 5, 16363 (2015)

25. Kohlrausch R. Theorie des elektrischen Rückstandes in der Leidner Flasche. Annalen der Physik und Chemie 91, 56-82, 179-213 (1854).

26. Hall D. O., Rao K. K. Photosynthesis (6th edn.). Cambridge University Press, 214 p. (1999).

27. Green B. R., Pichersky E., Kloppstech K. Chlorophyll a/b-binding proteins: an extended family. Trends in biochemical sciences 16, 181-186 (1991). 